\title{
The International Consortium on Lithium Genetics (ConLiGen): An Initiative by the NIMH and IGSLI to Study the Genetic Basis of Response to Lithium Treatment
}

\author{
Thomas G. Schulze ${ }^{a, g, \varepsilon} \quad$ Martin Aldam, ${ }^{m} \quad$ Mazda Adli ${ }^{\text {h, } \varepsilon} \quad$ Nirmala Akula $^{a}$ \\ Raffaella Ardau $^{\mathrm{t}}$ Elise T. Bui ${ }^{\mathrm{a}}$ Caterina Chillotti ${ }^{\mathrm{t}}$ Sven Cichon $^{\mathrm{i}, \zeta}$ Piotr Czerski ${ }^{\mathrm{v}}$ \\ Maria Del Zompo ${ }^{t, u}$ Sevilla D. Detera-Wadleigh ${ }^{a} \quad$ Paul Grof $^{n, o, \varepsilon} \quad$ Oliver Gruber $^{\mathrm{j}}$ \\ Ryota Hashimoto $^{x, \delta}$ Joanna Hauser ${ }^{v}$ Rebecca Hoban ${ }^{b, c}$ Nakao Iwata ${ }^{y, \delta}$ Layla Kassem ${ }^{a}$ \\ Tadafumi Kato $^{z, \delta}$ Sarah Kittel-Schneiderk Sebastian Kliwickiw John R. Kelsoe ${ }^{\text {b, } c}$ \\ Ichiro Kusumi ${ }^{\beta, \delta}$ Gonzalo Laje $^{a}$ Susan G. Leckband ${ }^{\text {b, d, e } \text { Mirko Manchia }^{\mathrm{u}} \text { Glenda MacQueen }}{ }^{\mathrm{p}}$ \\ Takuya Masui $^{\beta, \delta} \quad$ Norio Ozaki ${ }^{\gamma} \delta$ Roy H. Perlis ${ }^{f}$ Andrea Pfennigl, $\varepsilon$ Paola Piccardi ${ }^{\mathrm{L}}$ \\ Sara Richardson $^{\mathrm{a}}$ Guy Rouleau ${ }^{\mathrm{q}}$ Andreas Reifk Janusz K. Rybakowski ${ }^{w} \varepsilon$ Johanna Sasse ${ }^{l, \varepsilon}$ \\ Johannes Schumacher ${ }^{a, i} \quad$ Giovanni Severino $^{u} \quad$ Jordan W. Smoller ${ }^{f}$ Alessio Squassina ${ }^{u}$ \\ Gustavo Turecki $^{r} \quad$ L. Trevor Young ${ }^{\text {s, } \varepsilon}$ Takeo Yoshikawa ${ }^{\alpha, \delta} \quad$ Michael Bauer $^{l, \varepsilon}$ \\ Francis J. McMahon ${ }^{a}$
}

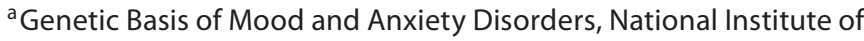
Mental Health, National Institutes of Health, Department of Health and Human Services, Bethesda, Md., b Department of Psychiatry, University of California San Diego, ' Department of Psychiatry, VA San Diego Healthcare System, and d Department of Pharmacy, VA San Diego Healthcare System, La Jolla, Calif., e'Skaggs School of Pharmacy and Pharmaceutical Sciences, University of California, San Diego, Calif., and ${ }^{\mathrm{f} D e p a r t m e n t}$ of Psychiatry, Massachusetts General Hospital and Harvard Medical School, Boston, Mass., USA; ${ }^{9}$ Department of Genetic Epidemiology in Psychiatry, Central Institute of Mental Health, Mannheim, ${ }^{\mathrm{h}}$ Department of Psychiatry and Psychotherapy, Charité - Universitätsmedizin Berlin, Campus Charité Mitte, Berlin, 'Department of Genomics, Life and Brain Center and Institute of Human Genetics, University of Bonn, Bonn, jDepartment of Psychiatry and Psychotherapy, Georg-August University, Göttingen, kDepartment of Psychiatry, Psychosomatics, and Psychotherapy, University of Würzburg, Würzburg, and 'Department of Psychiatry and Psychotherapy, University Hospital Carl Gustav Carus, Technische Universität Dresden, Dresden, Germany; ${ }^{m}$ Department of Psychiatry, Dalhousie University, Halifax, N.S., " Mood Disorders Center of Ottawa, Ottawa, Ont., 'Department of Psychiatry, University of Toronto, Toronto, Ont.,
\end{abstract}

PDepartment of Psychiatry, University of Calgary, Calgary, Alta., ${ }^{\mathrm{q} C H U}$ Sainte-Justine Research Center, Department of Medicine, University of Montreal, and 'Department of Psychiatry, Douglas Hospital Research Institute, McGill University, Montreal, Que., and ${ }^{\mathrm{S} D e p a r t m e n t}$ of Psychiatry, University of British Columbia, Vancouver, B.C., Canada; ${ }^{\mathrm{t} U n i t}$ of Clinical Pharmacology, Hospital University Agency, and 'Department of Neuroscience 'B.B. Brodie', University of Cagliari, Cagliari, Italy; ${ }^{\vee}$ Psychiatric Genetic Unit, and ${ }^{w}$ Department of Adult Psychiatry, Poznan University of Medical Sciences, Poznan, Poland; ${ }^{\times}$Osaka University Graduate School of Medicine, Osaka, ${ }^{y}$ Department of Psychiatry, Fujita Health University School of Medicine, Toyoake, ${ }^{\mathrm{L}}$ Laboratory for Molecular Dynamics of Mental Disorders, and ${ }^{\alpha}$ Laboratory for Molecular Psychiatry, RIKEN Brain Science Institute, Saitama, ${ }^{\beta}$ Department of Psychiatry, Hokkaido University Graduate School of Medicine, Sapporo, ${ }^{\gamma}$ Department of Psychiatry, Nagoya University Graduate School of Medicine, Nagoya, Japan; ${ }^{\delta}$ The Japanese Collaborative Group on the Genetics of Lithium Response in Bipolar Disorder; ${ }^{\varepsilon}$ The International Group for the Study of Lithium-Treated Patients (IGSLI); ' Institute of Neurosciences and Medicine (INM-1), Research Center Juelich, Juelich, Germany

\section{KARGER}

두 2010 S. Karger AG, Basel

Fax +41613061234 E-Mail karger@karger.ch www.karger.com www.karger.com/nps
Thomas G. Schulze, MD, Unit on the Genetic Basis of Mood and Anxiety Disorders National Institute of Mental Health (NIMH), National Institutes of Health (NIH) 35 Convent Drive, Bldg. 35, Rm 1A205, MSC 3719 Bethesda, MD 20892-3719 (USA)

Tel. +1 301451 7213, Fax +1 301402 9081, E-Mail schulzet@ mail.nih.gov 


\section{Key Words}

Manic-depressive illness $\cdot$ Schizoaffective disorder . Mood stabilizer - Antidepressants $\cdot$ Suicidal behavior . Genome-wide association study · Neurogenesis • Neuroplasticity

\begin{abstract}
For more than half a decade, lithium has been successfully used to treat bipolar disorder. Worldwide, it is considered the first-line mood stabilizer. Apart from its proven antimanic and prophylactic effects, considerable evidence also suggests an antisuicidal effect in affective disorders. Lithium is also effectively used to augment antidepressant drugs in the treatment of refractory major depressive episodes and prevent relapses in recurrent unipolar depression. In contrast to many psychiatric drugs, lithium has outlasted various pharmacotherapeutic 'fashions', and remains an indispensable element in contemporary psychopharmacology. Nevertheless, data from pharmacogenetic studies of lithium are comparatively sparse, and these studies are generally characterized by small sample sizes and varying definitions of response. Here, we present an international effort to elucidate the genetic underpinnings of lithium response in bipolar disorder. Following an initiative by the International Group for the Study of Lithium-Treated Patients (www.IGSLI.org) and the Unit on the Genetic Basis of Mood and Anxiety Disorders at the National Institute of Mental Health, lithium researchers from around the world have formed the Consortium on Lithium Genetics (www.ConLiGen.org) to establish the largest sample to date for genome-wide studies of lithium response in bipolar disorder, currently comprising more than 1,200 patients characterized for response to lithium treatment. A stringent phenotype definition of response is one of the hallmarks of this collaboration. ConLiGen invites all lithium researchers to join its efforts.
\end{abstract}

Copyright $\odot 2010$ S. Karger AG, Base

\section{Background}

The articles in this special issue of Neuropsychobiology comprehensively review the use of lithium as a mood stabilizer in bipolar and unipolar affective disorders. They show that 60 years after Cade's discovery, lithium is still a first-line choice for prophylaxis in bipolar disorder. They furthermore discuss the evidence regarding lithium's antisuicidal effects, its use as an augmentation strategy in the treatment of unipolar depression, and provide novel insights into its neurobiological mechanisms of ac- tion. Finally, current pharmacogenetic knowledge about lithium treatment is reviewed. Taken together, however, these articles also highlight that, despite decades of lithium use in psychiatry and despite the current emphasis on the study of psychiatric genetics in modern biological psychiatry, pharmacogenetic data regarding lithium treatment have a tendency to be circumstantial and inconclusive.

Pharmacogenetics is a rapidly growing field that holds considerable promise for the development of medications that are more personalized and effective than those currently available. In all areas of medicine, pharmacogenetic studies of outcomes such as treatment response or characteristic side effects are on the rise; based on these findings, more and more pharmacogenetic tests are being offered and approved by the US Food and Drug Administration [1]. Pretreatment genetic testing has now even been added to the prescribing information for the anticoagulant warfarin [2]. Similarly, the Food and Drug Administration updated labeling for carbamazepine, recommending that patients of Asian ancestry be screened for the presence of the HLA allele $B^{*} 1502$ that has been implicated in carbamazepine-induced Stevens-Johnson syndrome and toxic epidermal necrolysis in Han Chinese people [3].

Sufficiently large, well-characterized samples as well as effective and efficient collaboration between academia and the pharmaceutical industry are among the critical prerequisites for success in the field of pharmacogenetics $[4,5]$. Pharmacogenetic research in psychiatry has long been characterized by single lab efforts and small sample sizes. Only recently has our field witnessed large collaborative studies such as the Sequenced Treatment Alternatives to Relieve Depression (STAR*D) study (http://www. edc.pitt.edu/stard/) [6] in the United States, or the Genome-Based Therapeutic Drugs for Depression (GENDEP) project (http://gendep.iop.kcl.ac.uk) [7] in Europe, both of which study the pharmacogenetics of major depression. Indeed, the STAR ${ }^{*} \mathrm{D}$ and GENDEP projects have already generated several intriguing findings concerning the genetics of treatment response and side effects [8-14]. It is hoped that genome-wide association studies (GWAS) conducted in these and other samples will significantly increase our ability to guide the pharmacological treatment of psychiatric patients through the identification of genetic markers.

Notably, despite lithium's proven efficacy [15], to date there has been only one GWAS examining this 'pharmacological workhorse' of psychiatry [16]. In two cohorts encompassing more than 800 lithium-treated patients, 
multiple regions of interest were identified but none met the threshold for genome-wide significance. While intriguing, no adequately powered cohort yet exists to replicate and extend these findings. Here, we present a worldwide effort to address this situation: the international Consortium on Lithium Genetics (ConLiGen), spearheaded by researchers from the International Group for the Study of Lithium-Treated Patients (IGSLI) and the National Institute of Mental Health (NIMH).

\section{The International Group for the Study of Lithium-Treated Patients}

The IGSLI is an international group of scientists dedicated to lithium-related research, and its use in mental illness and mood disorders in particular. Founded in 1988 by Mogens Schou (Risskov/Aarhus, Denmark), Bruno Müller-Oerlinghausen (Berlin, Germany), and Paul Grof (Ottawa, Canada), the IGSLI has significantly contributed to lithium research over the past 20 years (www. igsli.org). Other scientists and centers have since joined the group, which currently comprises 35 members from Austria, Canada, the Czech Republic, Denmark, Germany, Poland, Switzerland, and the United States. The main goal of this group has been to conduct systematic work on those key questions regarding lithium treatment that can only be resolved by joint international effort. Unified designs have been created and scientific data from the IGSLI member centers have been linked for the purpose of shared analysis. This approach allows investigators to work with large numbers of prospectively followed patients - something that could only be accomplished via a multicenter approach. Overall, IGSLI research is based on shared, standardized, computer-based documentation of patients' diagnoses, family histories, course of illness before and during treatment, and on comparable modalities of treatment. The group meets regularly at research conferences to plan and discuss joint projects and to prepare publications.

At the 21st IGSLI meeting, which took place in late September 2007 in Dresden, Germany, the group discussed the results from the first, newly released GWAS of bipolar disorder, performed by researchers from the NIMH and Germany [17]. The strongest findings identified and replicated in this study were those encoding diacylglycerol kinase eta, a key protein in the lithium-sensitive phosphatidylinositol pathway and several genes in the Wnt-signaling cascade. Given the absence of a hypothesis-driven selection of single nucleotide polymor- phisms in GWAS - a method more typical of candidate gene association studies - the observation that these findings implicated pathways relevant to lithium's mechanism of action was particularly intriguing. Spurred on by these findings, the IGSLI researchers concluded that studying these genes in samples that included data on patient response to lithium treatment could improve our understanding of how these genes determine response to lithium treatment and impact susceptibility to bipolar disorder. The IGSLI collaborators thus agreed to explore a framework that would allow researchers to engage in genetic studies of lithium response that were sufficiently powered. It was stated that such an endeavor should allow for participation by all bona fide lithium researchers within and beyond the IGSLI, while maintaining the highest possible level of stringency regarding phenotype definition.

\section{May 6, 2008: \\ The Consortium on Lithium Genetics Is Born}

Following an invitation by IGSLI member Thomas G. Schulze and Francis J. McMahon, both from the NIMH's Unit on the Genetic Basis of Mood and Anxiety Disorders, prominent scientists in the field of lithium and bipolar genetic research met at the NIMH to discuss the possibility of creating an international consortium dedicated to the study of lithium pharmacogenetics. In attendance were (in alphabetical order): Martin Alda (Halifax, N.S., Canada), Michael Bauer (Dresden, Germany), Maria Del Zompo (via phone from Cagliari, Italy), Gonzalo Laje (Bethesda, Md., USA), Francis J. McMahon (Bethesda, Md., USA), Mirko Manchia (Cagliari, Italy), Roy H. Perlis (Boston, Mass., USA), Janusz K. Rybakowski (Poznan, Poland), Thomas G. Schulze (Bethesda, Md., USA), Johannes Schumacher (Bethesda, Md., USA), and Jordan W. Smoller (Boston, Mass., USA).

Reviewing evidence from the literature, and based on their own observations, the group emphasized the evident familiality in lithium treatment response, raising the possibility that genetic variation may contribute to interindividual differences in treatment response. If such differences could be identified, they might facilitate the development of novel treatments for bipolar disorder, or allow for better matching between patients and treatments. Over the last decade, the quest for a 'personalized medicine' approach in psychiatry has propelled a host of pharmacogenetic studies. Because of the lengthy trialand-error process that currently characterizes the search 
for the most optimal treatment, pharmacogenetic studies in psychiatry have traditionally focused on treatment response or adverse effects associated with antidepressants or antipsychotic medications [18-22]. While initially limited by small sample sizes, pharmacogenetic studies in psychiatry have increasingly come to rely on large-scale collaborative efforts, such as STAR ${ }^{*} \mathrm{D}$, GENDEP, or the Clinical Antipsychotic Trials of Intervention Effectiveness (CATIE) project. While some pharmacogenetic studies performed with these collaborative samples have produced intriguing results, difficulties in defining stringent target phenotypes across the various subsamples remain an important challenge $[23,24]$.

The researchers gathered at the NIMH on May 6, 2008 noted that, despite considerable and well-documented worldwide experience with lithium as an effective antimanic agent, mood stabilizer, and putative antisuicidal agent, there is a surprising dearth of large-scale pharmacogenetic studies of lithium treatment. We thus decided to create an international initiative whose goal would be to facilitate high-quality, well-powered analyses of lithium treatment response data that would ultimately allow for robust conclusions. The Consortium on Lithium Genetics, hereafter referred to as ConLiGen, was born.

\section{ConLiGen's Scientific Goals}

ConLiGen aims to identify genetic determinants of response to lithium treatment in bipolar disorder, as well as genetic determinants of adverse events emerging during lithium treatment. In the long run, ConLiGen may also study response to lithium treatment in general (e.g. lithium augmentation in the treatment of major depression).

\section{Membership in ConLiGen}

Any bona fide researcher or research group with access to samples of lithium-treated patients for whom DNA is available can join ConLiGen. Any new admission request is voted upon by ConLiGen members.

\section{Communication between the ConLiGen Members}

To ensure a constant exchange of ideas between members and allow for a straightforward realization of ConLiGen's goals, a monthly conference call is conducted.
Furthermore, members meet once or twice a year at international meetings of various biological psychiatric organizations.

\section{ConLiGen Advisory Board}

An Advisory Board comprising international experts in the field of mood disorders research, and lithium research in particular, was established to offer ConLiGen an outside perspective as well as guidance on broad scientific directions, to serve as a liaison to nonacademic communities such as funding institutions, or industry, and finally, to act as one of ConLiGen's publicly visible faces. Currently, the following researchers are members of the Advisory Board (in alphabetical order): Robert $\mathrm{H}$. Belmaker (Division of Psychiatry, Ben Gurion University of the Negev, Beersheva, Israel), Gian Luigi Gessa (Department of Neuroscience 'B.B. Brodie', University of Cagliari, Cagliari, Italy), Paul Greengard (Laboratory of Molecular and Cellular Neuroscience, Rockefeller University, New York, N.Y., USA), Kay R. Jamison (Department of Psychiatry and Behavioral Sciences, Johns Hopkins University School of Medicine, Baltimore, Md., USA), Richard S. Jope (Department of Psychiatry and Behavioral Neurobiology, University of Alabama at Birmingham, Birmingham, Ala., USA), Husseini K. Manji (CNS \& Pain, Johnson and Johnson Pharmaceutical Research and Development, Titusville, N.J., USA), and Leon E. Rosenberg (Department of Molecular Biology and the Woodrow Wilson School of Public and International Affairs, Princeton University, Princeton, N.J., USA).

\section{Phenotype Definition of Lithium Response: A Major Prerequisite for Pharmacogenetic Studies of Lithium}

ConLiGen's first and most crucial goal is to define the phenotype of lithium response. Treatment response is a complex construct that requires researchers to make judgments about adequacy of treatment and tolerability as well as assess changes in episode frequency or symptom severity. In many cases this information must be assessed retrospectively, with the inherent limitations associated with recall bias, missing information, or the fact that the treatment has not followed a strict research protocol. One scale that incorporates such data is an 11-point scale developed by Martin Alda and colleagues [25] 
Name:

Date:

\section{Criterion A}

The criterion $\mathrm{A}$ is used to determine an association between clinical improvement and the treatment. The rating should apply to the period of treatment considered adequate in duration and dosage. The illness activity should be judged by frequency, severity, and duration of episodes.

$10=$ Complete response, no recurrences in the course of adequate treatment, no residual symptoms, and full functional recovery

$9=$ Very good response, no recurrences, but the patient may have minimal residual symptoms (transient anxiety, sleep disturbance, dysphoria, irritability) not requiring any intervention

$8=\quad$ Very good response. Illness activity reduced by more than $90 \%$

$7=\quad$ Good response. Illness activity reduced by $80-90 \%$

$6=$ Good response. Reduction in activity of illness by $65-80 \%$

$5=\quad$ Moderate response. Reduction in illness activity by $50-65 \%$

$4=\quad$ Moderate improvement. Reduction in illness activity by $35-50 \%$

$3=$ Mild improvement. Reduction of illness activity by $20-35 \%$

$2=\quad$ Mild improvement. Reduction of illness activity by $10-20 \%$

$1=\quad$ Minimal improvement. Reduction of illness activity by $0-10 \%$

$0=\quad$ No change or worsening

\section{A Criterion Score:}

\section{Criteria B}

The criteria B are used to establish whether there is a causal relationship between clinical improvement and the treatment. Score 0 , 1 or 2 points for each item:

\section{B1: Number of episodes off the treatment.}

$$
\begin{aligned}
& 0=4 \text { or more episodes } \\
& 1=\quad 2 \text { or } 3 \text { episodes } \\
& 2=\quad 1 \text { episode }
\end{aligned}
$$

B1:

(C) Martin Alda, 2002
Drug: __ Evaluated By:

\section{B2: Frequency of episodes off the treatment.}

$0=\quad$ Average to high, including rapid cycling

$1=$ Low, spontaneous remissions of 3 or more years on average

$2=1$ episode only, risk of recurrence cannot be established

B2:

B3: Duration of the treatment.

$0=2$ or more years

$1=1-2$ years

$2=$ Less than 1 year

B3:

B4: Compliance during period(s) of stability.

$0=\quad$ Excellent, e.g. documented by drug levels in the therapeutic range

$1=$ Good, more than 80\% levels in the therapeutic range

$2=$ Poor, repeatedly off treatment, less than $80 \%$ levels in the therapeutic range

B4:

B5: Use of additional medication during the period of stability.

$0=\quad$ None except infrequent sleep medication (1 per week or less); no other mood stabilizers, antidepressants or antipsychotics for control of mood symptoms

$1=$ Low-dose antidepressants or antipsychotics as an "insurance", or prolonged use of sleep medication

$2=$ Prolonged or systematic use of an antidepressant or antipsychotic

B5:

\section{B Criteria Score:}

Total Scale Score:

(Subtract B from A)

Fig. 1. Retrospective criteria of long-term treatment response in research subjects with bipolar disorder.

(fig. 1); other approaches include longitudinal outcome measures that consider time to recurrence or symptom burden during treatment $[16,26]$.

The 11-point scale measures the extent of improvement during long-term treatment. The scale's A score is a composite measure of change in frequency, duration, and severity of illness episodes in the course of lithium treatment. It is weighted by factors that influence the degree to which the observed clinical change is considered to be due to lithium (B1-B5 scores in the scale). The scale has been developed in the context of a study assessing response to treatment in subjects not followed according to a research protocol, namely relatives of probands in our genetic studies [25]. Subsequently, it has been widely used in several other studies at IGSLI centers [27-29] and at other centers involved in lithium research [pers. commun. from John Kelsoe, San Diego, Calif., USA and Maria del Zompo, Cagliari, Italy], which imparts face validity. Within ConLiGen, phenotypic assessment will be based on any available information in- 
cluding life charts when available and quantified using the scale; interrater reliability meetings will be organized, facilitated by ConLiGen member Martin Alda, and case vignettes will also be reviewed to establish between-center reliability.

Variables describing treatment tolerability or side effects may be studied in subsequent projects. Because the issue of 'best response phenotype' is far from trivial, ConLiGen will strive to continuously weigh evidence from future clinical and biological studies of lithium in an effort to refine the definition of phenotype response. Evaluating response to long-term treatment in an illness with a highly variable natural course presents a challenge. Many patients with bipolar disorder experience spontaneous remissions of variable timing and duration. Moreover, in a pharmacogenetic study we need to evaluate the quality of response not for groups of subjects as in clinical trials but individually for each patient. While prospective studies will be able to implement more precise measures, our approach is a practical way to assess the quality of response in a variety of patients treated in diverse settings.

\section{ConLiGen's Current Project and Long-Term Mission}

ConLiGen is poised to assess all aspects of the pharmacogenetics of lithium treatment in psychiatric disorders, including the study of genetic susceptibility to potential treatment-emergent adverse events (e.g. weight gain, hypothyroidism, tremor). As its first project, ConLiGen intends to conduct a GWAS of stringently defined response to lithium treatment in bipolar disorder. ConLiGen members and the various research centers which they are affiliated with are joining their samples for a centralized genotyping effort to be performed at the Unit on the Genetic Basis of Mood and Anxiety Disorders of the NIMH and the Department of Genomics of the Life and Brain Center at the University of Bonn, Germany. For the primary projects, a previously validated scale will be used to define response to lithium treatment, as described above. Individuals scoring between 7 and 10 will be considered lithium 'responders', while individuals with scores between 0 and 6 will be considered 'nonresponders'. Presently, the total sample comprises more than 1,200 bipolar patients for whom response to lithium treatment has been or is currently being assessed by means of the scale. From preliminary analyses conducted in select IGSLI samples (data not shown), we can assume that about $35-40 \%$ of patients will qualify as responders. Previous studies $[8,9]$ suggest larger genetic effect sizes (e.g. allelic odds ratios between 1.5 and 2) for a narrowly defined pharmacogenetic phenotype than for a categorically defined clinical diagnosis. Thus, assuming a minor allele frequency of 0.3 and genotype relative risks of 1.4 for individuals heterozygous, and of 1.96 for individuals homozygous for the risk allele, the combined ConLiGen sample will have a power of $83 \%$ to detect an effect at a significance level of $1 \times 10^{-8}$ [30].

Although the combined ConLiGen sample will be the largest sample to date to investigate lithium response on a genome-wide scale, we are aware that any finding, regardless of whether it reaches levels of genome-wide significance, will ultimately have to be confirmed in independent samples. Thus, ConLiGen's mission will not be finished after the completion of its GWAS. On the contrary, ConLiGen will continue to invite researchers to join its efforts in order to increase the available sample size of patients adequately characterized for lithium response. In collaboration with both IGSLI centers and large, long-standing multicenter projects such as the NIMH Bipolar Disorder Genetics Initiative, ConLiGen will be actively engaged in supporting and organizing urgently needed prospective studies of lithium response in bipolar disorder and other conditions.

Since Cade discovered lithium's beneficial effects in the treatment of bipolar disorder 60 years ago, this agent has become almost synonymous with the treatment of bipolar disorder worldwide [15]. Yet, little is known about the genetic underpinnings of lithium response or the development of side effects associated with its use. In a scientific environment characterized by calls for personalized medicine and the growth of large-scale pharmacogenetic studies in many fields of medicine, ConLiGen's goal is to put lithium at the forefront of pharmacogenetic studies in psychiatry.

\section{Acknowledgments}

This research was in part supported by the Intramural Program of the NIMH. Dr. Thomas G. Schulze's work is furthermore supported by NARSAD. Dr. Martin Alda's work on the phenotype of lithium response has been supported by grants from the Canadian Institutes of Health Research (CIHR, grant 64410) and by NARSAD. Dr. Smoller's work is supported in part by a grant from the NIMH (MH079799). Dr. Reif is supported by the Deutsche Forschungsgemeinschaft (DFG) (KFO 125 and SFB TRR 58) and the German Ministry for Education and Research (BMBF) (IZKF N-4). The work of the Cagliari group was partly supported by the Regional Councillorship of Health, 'Regione Autonoma della Sardegna'. 


\section{References}

$\checkmark 1$ Swen JJ, Huizinga TW, Gelderblom H, de Vries EG, Assendelft WJ, Kirchheiner J, Guchelaar HJ: Translating pharmacogenomics: challenges on the road to the clinic. PLoS Med 2007;4:e209.

-2 Wadelius M, Sörlin K, Wallerman O, Karlsson J, Yue QY, Magnusson PK, Wadelius C, Melhus $\mathrm{H}$ : Warfarin sensitivity related to CYP2C9, CYP3A5, ABCB1 (MDR1) and other factors. Pharmacogenomics J 2004;4:4048.

>3 Chung WH, Hung SI, Hong HS, Hsih MS, Yang LC, Ho HC, Wu JY, Chen YT: Medical genetics: a marker for Stevens-Johnson syndrome. Nature 2004;428:486.

4 Penny MA, McHale D: Pharmacogenomics and the drug discovery pipeline: when should it be implemented? Am J Pharmacogenomics 2005;5:53-62.

$\checkmark 5$ Roses AD: Pharmacogenetics in drug discovery and development: a translational perspective. Nat Rev Drug Discov 2008;7:807817.

-6 Fava M, Rush AJ, Trivedi MH, Nierenberg AA, Thase ME, Sackeim HA, Quitkin FM, Wisniewski S, Lavori PW, Rosenbaum JF, Kupfer DJ: Background and rationale for the sequenced treatment alternatives to relieve depression (STAR*D) study. Psychiatr Clin North Am 2003;26:457-494.

7 Uher R, Farmer A, Maier W, Rietschel M, Hauser J, Marusic A, Mors O, Elkin A, Williamson RJ, Schmael C, Henigsberg N, Perez J, Mendlewicz J, Janzing JG, Zobel A, Skibinska M, Kozel D, Stamp AS, Bajs M, Placentino A, Barreto M, McGuffin P, Aitchison KJ: Measuring depression: comparison and integration of three scales in the GENDEP study. Psychol Med 2008;38:289-300.

$\checkmark 8$ McMahon FJ, Buervenich S, Charney D, Lipsky R, Rush AJ, Wilson AF, Sorant AJ, Papanicolaou GJ, Laje G, Fava M, Trivedi $\mathrm{MH}$, Wisniewski SR, Manji H: Variation in the gene encoding the serotonin $2 \mathrm{~A}$ receptor is associated with outcome of antidepressant treatment. Am J Hum Genet 2006;78:804814.

-9 Laje G, Paddock S, Manji H, Rush AJ, Wilson AF, Charney D, McMahon FJ: Genetic markers of suicidal ideation emerging during citalopram treatment of major depression. Am J Psychiatry 2007;164:1530-1538.

10 Perlis RH, Purcell S, Fava M, Fagerness J, Rush AJ, Trivedi MH, Smoller JW: Association between treatment-emergent suicidal ideation with citalopram and polymorphisms near cyclic adenosine monophosphate response element binding protein in the STAR*D study. Arch Gen Psychiatry 2007;64:689-697.
1 Paddock S, Laje G, Charney D, Rush AJ, Wilson AF, Sorant AJ, Lipsky R, Wisniewski SR, Manji H, McMahon FJ: Association of GRIK4 with outcome of antidepressant treatment in the STAR*D cohort. Am J Psychiatry 2007;164:1181-1188.

12 Perlis RH, Moorjani P, Fagerness J, Purcell S, Trivedi MH, Fava M, Rush AJ, Smoller JW: Pharmacogenetic analysis of genes implicated in rodent models of antidepressant response: association of TREK1 and treatment resistance in the STAR $\left(^{*}\right)$ D study. Neuropsychopharmacology 2008;33:2810-2819.

13 Lekman M, Laje G, Charney D, Rush AJ Wilson AF, Sorant AJ, Lipsky R, Wisniewski SR, Manji H, McMahon FJ, Paddock S: The FKBP5-gene in depression and treatment response - an association study in the Sequenced Treatment Alternatives to Relieve Depression (STAR*D) Cohort. Biol Psychiatry 2008;63:1103-1110.

14 Uher R, Huezo-Diaz P, Perroud N, Smith R, Rietschel M, Mors O, Hauser J, Maier W, Kozel D, Henigsberg N, Barreto M, Placentino A, Dernovsek MZ, Schulze TG, Kalember P, Zobel A, Czerski PM, Larsen ER, Sourey D, Giovannini C, Gray JM, Lewis CM, Farmer A, Aitchison KJ, McGuffin P, Craig I: Genetic predictors of response to antidepressants in the GENDEP project. Pharmacogenomics J 2009;9:225-233.

15 Bauer M, Grof P, Müller-Oerlinghausen B (eds): Lithium in Neuropsychiatry: The Comprehensive Guide. London, Informa Healthcare, 2006.

16 Perlis RH, Smoller JW, Ferreira MAR, Mc Quillin A, Bass N, Chir MB, Lawrence J, Sachs GS, Nimgaonkar V, Scolnick EM, Gurling H, Sklar P, Purcell S: A genomewide association study of response to lithium for prevention of recurrence in bipolar disorder. Am J Psychiatry 2009;166:718-725

17 Baum AE, Akula N, Cabanero M, Cardona I, Corona W, Klemens B, Schulze TG, Cichon S, Rietschel M, Nöthen MM, Georgi A Schumacher J, Schwarz M, Abou Jamra R, Höfels S, Propping P, Satagopan J, DeteraWadleigh SD, Hardy J, McMahon FJ: A genome-wide association study implicates diacylglycerol kinase eta (DGKH) and several other genes in the etiology of bipolar disorder. Mol Psychiatry 2008;13:197-207.

18 Rietschel M, Kennedy JL, Macciardi F, Meltzer HY: Application of pharmacogenetics to psychotic disorders: the first consensus conference. The Consensus Group for Outcome Measures in Psychoses for Pharmacological Studies. Schizophr Res 1999;37:191-196.
19 Lerer B, Segman RH, Fangerau H, Daly AK, Basile VS, Cavallaro R, Aschauer HN, McCreadie RG, Ohlraun S, Ferrier N, Masellis M, Verga M, Scharfetter J, Rietschel M, Lovlie R, Levy UH, Meltzer HY, Kennedy JL, Steen VM, Macciardi F: Pharmacogenetics of tardive dyskinesia: combined analysis of 780 patients supports association with dopamine $\mathrm{D}_{3}$ receptor gene Ser9Gly polymorphism. Neuropsychopharmacology 2002;27:105-119.

20 Malhotra AK, Murphy GM Jr, Kennedy JL: Pharmacogenetics of psychotropic drug response. Am J Psychiatry 2004;161:780-796.

21 Correll CU, Malhotra AK: Pharmacogenetics of antipsychotic-induced weight gain. Psychopharmacology (Berl) 2004:477-489.

-22 Malhotra AK, Lencz T, Correll CU, Kane JM: Genomics and the future of pharmacotherapy in psychiatry. Int Rev Psychiatry 2007;19: 523-530.

$\checkmark 23$ van den Oord EJ, Adkins DE, McClay J, Lieberman J, Sullivan PF: A systematic method for estimating individual responses to treatment with antipsychotics in CATIE. Schizophr Res 2009; 107:13-21.

24 Need AC, Keefe RS, Ge D, Grossman I, Dickson S, McEvoy JP, Goldstein DB: Pharmacogenetics of antipsychotic response in the CATIE trial: a candidate gene analysis. Eur J Hum Genet 2009; 17:946-957.

-25 Grof P, Duffy A, Cavazzoni P, Grof E, Garnham J, MacDougall M, O’Donovan C, Alda $\mathrm{M}$ : Is response to prophylactic lithium a familial trait? J Clin Psychiatry 2002;63: 942-947.

26 Perlis RH, Ostacher MJ, Patel JK, Marangell LB, Zhang H, Wisniewski SR, Ketter TA, Miklowitz DJ, Otto MW, Gyulai L, ReillyHarrington NA, Nierenberg AA, Sachs GS, Thase ME: Predictors of recurrence in bipolar disorder: primary outcomes from the Systematic Treatment Enhancement Program for Bipolar Disorder (STEP-BD). Am J Psychiatry 2006;163:217-224.

27 Garnham J, Munro A, Slaney C, Macdougall M, Passmore M, Duffy A, O’Donovan C, Teehan A, Alda M: Prophylactic treatment response in bipolar disorder: results of a naturalistic observation study. J Affect Disord 2007; 104:185-190.

28 Passmore MJ, Garnham J, Duffy A, MacDougall M, Munro A, Slaney C, Teehan A, Alda M: Phenotypic spectra of bipolar disorder in responders to lithium versus lamotrigine. Bipolar Disord 2003;5:110-114.

29 Hajek T, Hahn M, Slaney C, Garnham J, Green J, Ruzickova M, Zvolsky P, Alda M: Rapid cycling bipolar disorders in primary and tertiary-care treated patients. Bipolar Disord 2008;10:495-502.

-30 Purcell S, Cherny SS, Sham PC: Genetic Power Calculator: design of linkage and association genetic mapping studies of complex traits. Bioinformatics 2003;19:149-150. 